\title{
Comunicación
}

\section{Frecuencia de piometra en perras pacientes de la Clínica de Ani- males Menores de la Facultad de Medicina Veterinaria de la Universidad Nacional Mayor de San Marcos durante el periodo 2009-2013}

\author{
Frequency of pyometra in bitches patients of the Small Animals Clinic of the \\ Faculty of Veterinary Medicine of Universidad Nacional Mayor de San Marcos \\ during the period 2009-2013
}

Norelli Solano C. ${ }^{1}$, Jacqueline Cahua U. ${ }^{1}$, Armando Gonzáles Z. ${ }^{2}$, César Gavidia C. ${ }^{2}$

\section{Resumen}

Se revisaron las fichas clínicas de perras mayores de seis meses que fueron atendidas en la Clínica de Animales Menores de la Facultad de Medicina Veterinaria de la Universidad Nacional Mayor de San Marcos durante el periodo 2009-2013, con el fin de obtener información estadística acerca de la frecuencia de piometra y su relación con la edad, raza, tamaño y estación del año. De los 4715 registros de perras, 207 fueron diagnosticados con piometra, representando un $4.4 \pm 0.6 \%$ de incidencia. Se halló asociación estadística entre la raza y la piometra, más no así con el tamaño del animal ni la estación del año.

Palabras clave: piometra; perras; frecuencia; edad; peso

\section{AbSTRaCT}

Clinical records of bitches older than six months that were treated at the Small Animals Clinic of the Faculty of Veterinary Medicine of the Universidad Nacional Mayor de San Marcos during the period 2009-2013 were revised to obtain statistical information about

${ }^{1}$ Clínica de Animales Menores, Facultad de Medicina Veterinaria, Universidad Nacional Mayor de San Marcos, Lima, Perú

${ }^{2}$ Laboratorio de Medicina Veterinaria Preventiva, Facultad de Medicina Veterinaria, Universidad Nacional Mayor de San Marcos, Lima, Perú

${ }^{3}$ E-mail: jcahuau@unmsm.edu.pe 
the frequency of pyometra and its relationship with age, breed, size and season of the year. Out of 4715 records of bitches, 207 were diagnosed with pyometra, representing an incidence of $4.4 \pm 0.6 \%$. A statistical association was found between pyometra and breed, but not with the size of the animal or the season of the year.

Key words: pyometra; bitches; frequency; age; weight

\section{INTRODUCCIÓN}

La piometra es una enfermedad que afecta comúnmente el aparato reproductor de las perras. Es también conocida como piometritis, endometritis catarral, endometritis quística crónica y complejo piometra. Esta patología es más común en hembras de 4 a 10 años, pero con menor predisposición en perras multíparas (Niskanen y Thrusfield, 1998). Esta patología puede clasificarse en aguda o crónica. Así mismo, si hay presencia de estrógenos el cérvix permanecerá abierto, disminuyendo el grado de intoxicación, pero si predomina la progesterona el cérvix permanecerá cerrado y se presentaría septicemia y muerte del animal (Morrow, 1986).

Clínicamente, las perras llegan a presentar inapetencia, depresión, fiebre, polidipsia y poliuria, diarreas, vómitos, abdomen distendido y secreción vaginal. También llegan a presentar deshidratación, hipoglucemia, disfunción hepática y renal (Fossum, 1999; Feldman y Nelson, 2007). En la historia clínica, lo más resaltante es que haya presentado celo en los últimos 60 a 90 días (Welch, 2007). El diagnóstico se basa en la historia clínica del paciente y de los signos clínicos que presente. También se puede recurrir a las pruebas diagnósticas, como son la radiografía, la ecografía, hemograma, química sanguínea, uroanálisis y citología vaginal (Fossum, 1999; Davidson, 1995; Nelson y Couto, 2010). El tratamiento más utilizado es la ovariohisterectomía, previa estabilización del paciente (Feldman y Nelson, 2007).
En el Perú no existen estudios sobre la frecuencia de la piometra, ni posibles relaciones con la edad, peso, raza y estación del año. Por otro lado, Niskanen y Thrusfield (1998) reportan 6\% de incidencia de piometra en la cuidad de Helsinki, Finlandia; Silva y Loaiza (2007) reporta 5\% entre los casos atendidos en la Clínica Veterinaria de la Universidad de Caldas, Colombia; así mismo, Fukuda (2001) relacionó la ocurrencia de piometra con tratamientos hormonales y edad de perras Beagle en Japón, y Jayaprakash et al. (2007) reportó una asociación directa entre piometra con la edad, raza y tamaño de la perra en la India.

El presente estudio determinó la frecuencia de presentación de casos de piometra en perras atendidas en un periodo de cinco años en una clínica veterinaria universitaria de Lima, Perú.

\section{MATERIALES y Métodos}

Se trabajó con las historias clínicas de pacientes caninos, enteras, hembras mayores de seis meses de edad atendidas en la la Clínica de Animales Menores de la Facultad de Medicina Veterinaria de la Universidad Nacional Mayor de San Marcos (FMVUNMSM) durante el periodo 2009-2013, y se identificó aquellas con diagnóstico clínico de piometra.

Los datos registrados corresponden a la edad ( $<1,1-4,5-8,9-12 ;>13$ años), raza (definidas, cruzadas o no definidas), tamaño 
Cuadro 1. Frecuencia de piometra en perras según la raza ${ }^{1}$

\begin{tabular}{lccc}
\hline Raza & Animales $(\mathrm{n})$ & Piometra $(\mathrm{n})$ & $\% \pm \mathrm{IC}^{2}$ \\
\hline Pitbull & 69 & 10 & $14.5 \pm 0.06$ \\
Fox Terrier & 50 & 7 & $14.0 \pm 0.07$ \\
Siberian Husky & 84 & 9 & $10.7 \pm 0.05$ \\
Pastor Alemán & 81 & 8 & $9.9 \pm 0.05$ \\
Cruce x Pequinés & 47 & 4 & $8.5 \pm 0.06$ \\
Pequinés & 300 & 23 & $7.7 \pm 0.02$ \\
Golden Retriever & 80 & 4 & $5.0 \pm 0.03$ \\
Schnauzer & 328 & 16 & $4.9 \pm 0.02$ \\
Cruzada (C) & 1283 & 58 & $4.5 \pm 0.01$ \\
Cocker Spaniel & 317 & 11 & $3.5 \pm 0.01$ \\
\hline
\end{tabular}

${ }^{1}$ Las 10 razas de mayor frecuencia

${ }^{2}$ Intervalo de confianza del $95 \%$

$(<10,11-20,>20 \mathrm{~kg})$ y estación del año (primavera, verano, otoño, invierno). La frecuencia de piometra se presentó en forma porcentual con intervalos de confianza al $95 \%$ y la relación estadística con las variables en estudio se analizó con la prueba de regresión logística.

\section{Resultados}

Se revisaron 4715 historias de pacientes hembras mayores de 6 meses correspondientes al periodo 2009-2013, de las cuales 207 presentaron piometra, lo cual representa $4.4 \pm 0.6 \%$ de frecuencia. La mayor proporción se encontró en perras Pitbull (14.5 \pm $0.1 \%)$, seguida por el Fox Terrier $(14.0 \pm$ $0.1 \%)$ y Siberian Husky $(10.7 \pm 0.1 \%)$. El análisis de regresión logística demostró que la raza representaba un factor de riesgo de presentación de piometra $(\mathrm{p}<0.05)$ (Cuadro 1).

La frecuencia de piometras según la edad y tamaño de las perras y estación del año se presenta en el Cuadro 2. La proporción de casos de piometra aumentó en fun- ción a la edad, observándose una mayor frecuencia en el grupo etario de 9 a 12 años $(\mathrm{p}<0.05)$. Así mismo, las perras de tamaño grande $(>20 \mathrm{~kg})$ presentaron la mayor frecuencia de piometras $(5.1 \pm 0.01 \%)$. Sin embargo, no se encontró una asociación significativa entre piometra y estación del año.

\section{Discusión}

La frecuencia de piometra de $4.4 \pm$ $0.6 \%$ para la población total de perras fue similar con la frecuencia de $4.8 \%$ reportado por Silva y Loaiza (2007) en el Hospital Veterinario de la Universidad de Caldas, Colombia (2004). Por otro lado, fue ligeramente menor al 6\% reportado por Niskanen y Thrusfield (1998) de casos atendidos en clínicas de Helsinki, Finlandia.

Las razas con mayor frecuencia de piometra en el presente estudio difieren de las razas Pastor Belga y Chow Chow reportadas por Niskanen y Thrusfield (1998) y de las razas Spitz, Doberman, Pastor Alemán, cruzados y Labrador reportadas por 
Cuadro 2. Frecuencia de piometras según la edad y tamaño de las perras y estación del año

\begin{tabular}{llccc}
\hline Variables & & $\begin{array}{c}\text { Observaciones } \\
(\mathrm{n})\end{array}$ & $\begin{array}{c}\text { Número de } \\
\text { casos }\end{array}$ & $\begin{array}{c}\text { Media } \pm \mathrm{IC} \\
95 \%\end{array}$ \\
\hline Edad (años) & $>1$ & 395 & 1 & $0.3 \pm 0.00$ \\
& $1-4$ & 1,719 & 33 & $1.9 \pm 0.006$ \\
& $5-8$ & 1,294 & 77 & $6.0 \pm 0.013$ \\
& $9-12$ & 972 & 80 & $8.2 \pm 0.017$ \\
Tamaño & $>12$ & 335 & 16 & $4.8 \pm 0.023$ \\
& Pequeño $(<10 \mathrm{~kg})$ & 2,275 & 101 & $4.4 \pm 0.01$ \\
& Mediano $(10-20 \mathrm{~kg})$ & 1,243 & 45 & $3.6 \pm 0.01$ \\
Estación del & Grande $(>20 \mathrm{~kg})$ & 1,197 & 61 & $5.1 \pm 0.01$ \\
año & Invierno & 1,324 & 52 & $3.9 \pm 0.010$ \\
& Otoño & 1,324 & 61 & $4.6 \pm 0.011$ \\
& Primavera & 728 & 27 & $3.7 \pm 0.014$ \\
& Verano & 1,339 & 67 & $5.0 \pm 0.012$ \\
\hline
\end{tabular}

Jayaprakash et al. (2007). Estas diferencias se deben principalmente a la frecuencia de perros de determinadas razas en cada país, pues dependen del desarrollo económico, moda y forma de vida de los propietarios.

El análisis estadístico para la variable edad indicó una tendencia de aumento en la frecuencia de piometra a medida que la edad se incrementa; es decir, a mayor edad del animal mayor predisposición a que presente piometra. Estos resultados concuerdan con otros reportes de la literatura (Niskanen y Thrusfield, 1998; Chastian et al., 1999; Egenvall et al., 2001; Fukuda, 2001; Jayaprakash et al., 2007). Asimismo, se indica que la piometra se presenta con mayor frecuencia en perras de mediana edad ( $>6$ años), dada la mayor estimulación de progesterona a través de los años (Fossum, 1999; Feldman y Nelson, 2007). Por otra parte, es necesario mencionar que existen casos de piometra en perras con edades de dos años, lo cual podría deberse a que los animales han recibido estrógenos o progestágenos exógenos en varias ocasiones, ya sea para evitar la preñez o el celo (Niskanen y Thrus- field, 1998; Fossum, 1999; Feldman y Nelson, 2007).

El tamaño no influyó en la presentación de la enfermedad, aunque las perras con más de 20 kilos presentaron la mayor frecuencia $(5.1 \pm 0.01 \%)$. Niskanen y Thrusfield (1998) halló una mayor frecuencia en perras de razas grandes; en cambio, Jayaprakash et al. (2007) encontró una mayor incidencia en perras de raza pequeñas, posiblemente debido a la mayor frecuencia de este tipo de perra en la ciudad en estudio.

\section{Literatura Citada}

1. Chastain CB, Panciera D, Waters $C$. 1999. Associations between age, parity, hormonal therapy and breed, and pyometra in Finnish dogs. Small Anim Endocrinol 9: 8.

2. Davidson P. 1995. Terapéutica veterinaria de pequeños animales. $12^{\circ} \mathrm{ed}$. España: Interamericana McGraw-Hill. p 1081-1083. 
3. Egenvall A, Hagman R, Bonnett BN, Hedhammar A, Olson P, Lagerstedt $A S$. 2001. Breed risk of pyometra in insured dog in Sweden. J Vet Intern Med 15: 530-538.

4. Feldman C, Nelson W. 2007. Endocrinología y reproducción en perros y gatos. $3^{\circ}$ ed. Buenos Aires, Argentina: McGraw-Hill Interamericana. $1234 \mathrm{p}$.

5. Fukuda S. 2001. Incidence of pyometra in colony- raised Beagle dogs. Exp Anim 50: 325-329.

6. Fossum T. 1999. Cirugía en pequeños animales. Buenos Aires, Argentina: Inter médica. $1282 \mathrm{p}$.

7. Jayaprakash R, Sathiamoorthy T, Sureshkumar R. 2007. Incidence of pyometra in bitches: a retrospective study of 249 cases. Madras. Tamilnadu J Vet Anim Sci 3: 164-165.
8. Morrow A. 1986. Current therapy in theriogenology: diagnosis, treatment and prevention of reproductive disease in small and large animals. $2^{\text {nd }}$ ed. Philadelphia, USA: WB Saunders. 1143 p.

9. Nelson R, Couto G 2010. Medicina interna de animales pequeños. $4^{\circ}$ ed. Buenos Aires, Argentina: Intermédica. $1504 \mathrm{p}$.

10. Niskanen M, Thrusfield M. 1998. Associations between age, parity, hormonal therapy and breed, and pyometra in finnish dogs. Vet Rec 143: 493-498.

11. Silva-Molano R, Loaiza-Echeverri A. 2007. Piometra en animales pequeños. Vet Zootec 1(2): 71-86.

12. Welch T. 2007. Cirugía en pequeños animales. $2^{\circ}$ ed. Buenos Aires, Argentina: Intermédica. $1632 \mathrm{p}$. 